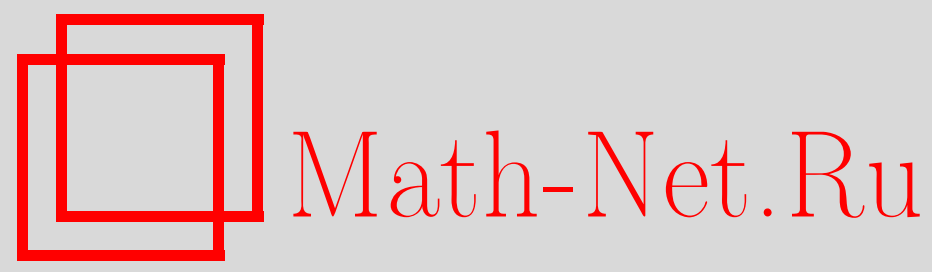

А. В. Рыжкова, Е. И. Яковлев, Расслоения с группами многозначных автоморфизмов, Матем. заметки, 2005, том 77, выпуск 4, 600-616

DOI: https://doi.org/10.4213/mzm2521

Использование Общероссийского математического портала Math-Net.Ru подразумевает, что вы прочитали и согласны с пользовательским соглашением http://www.mathnet.ru/rus/agreement

Параметры загрузки:

IP: 3.93 .64 .190

26 апреля 2023 г., $17: 44: 49$ 


\title{
РАССЛОЕНИЯ С ГРУППАМИ МНОГОЗНАЧНЫХ АВТОМОРФИЗМОВ
}

\author{
А. В. Рыжкова, Е.И. Яковлев
}

Построена категория, объектами которой являются главные расслоения с фиксированной базой - гладким многообразием $B$, структурной группой $T^{k}$ и конечной группой $\Delta$ многозначных автоморфизмов; от морфизмов требуется эквивариантность относительно $\Delta$. Найдены инварианты, с их помощью вычислена группа классов эквивалентности объектов категории. Приведены примеры, указаны приложения к динамике систем с гироскопическими силами.

Библиография: 8 названий.

Как известно, анализ ряда задач математической физики приводит к рассмотрению гироскопических систем с многозначным функционалом действия [1]. В случае конечности индекса иррациональности $k-1$ формы гироскопических сил такой системы преодолеть некоторые из возникающих трудностей удается с помощью главного расслоения $\xi$, для которого базой является конфигурационное многообразие $B$, а структурной группой - тор $T^{k}[2]$, [3]. Предположим, что интересующая нас гироскопическая система обладает конечной группой симметрий $\Delta$, т.е. инвариантна относительно некоторого действия $R: B \times \Delta \rightarrow B$. Возникает вопрос - какими дополнительными свойствами должно при этом обладать соответствующее расслоение $\xi$ ?

Нами получен точный ответ на этот вопрос. Оказывается, что на тотальном пространстве $E$ индуцируется многозначное действие групшы $\Delta$. При этом $\Delta$ становится группой многозначных автоморфизмов расслоения, поэтому мы назьваем $\xi$ почти $\Delta$-расслоением. Настоящая работа посвящена построению и исследованию категории почти $\Delta$-расслоений. Основной результат состоит в нахождении инвариантов и вычислении с их помощью группы классов эквивалентности почти $\Delta$-расслоений с заданной базой $B$ и фиксированными $T^{k}, \Delta$ и $R$.

В [4] одним из авторов данной работы с несколько иной точки зрения рассматривались почти $\Delta$-расслоения, для которых действие группы $\Delta$ на базе $B$ свободно. В этой ситуации пространство орбит $B / \Delta$ является гладким многообразием, а фактор-отображение $\nu: B \rightarrow B / \Delta$ - регулярным накрытием. Если $p: E \rightarrow B$-проекция расслоения $\xi$, то $\nu \circ p: E \rightarrow B / \Delta$ - проекция локально-тривиального расслоения со стандартным слоем $\Delta \times T^{k}$ и многозначными функциями перехода со значениями в той же групе $\Delta \times T^{k}$. Такие расслоения названы в [4] почти главными.

Работа выполнена при финансовой поддержке Российского фонда фундаментальных исследований, грант № 01-01-00590. 
В данной работе действие группы $\Delta$ на $B$ предполагается произвольньм, что существенно расширяет множество объектов изучаемой категории (см. примеры в п. 5). Кроме того, инварианты почти $\Delta$-расслоений здесь строятся в терминах гомологий базы $B, \mathrm{a}$ не пространства орбит $B / \Delta$ как в [4].

1. Категория почти $\Delta$-расслоений. Пусть $\xi$-гладкое главное расслоение с проекцией $p: E \rightarrow B$ и структурной группой $T^{k}=(\mathbb{R} / \mathbb{Z})^{k}$. Рассмотрим конечную групу $\Delta$ и правое действие $R: B \times \Delta \rightarrow B$. Как обычно, для $a \in B$ и $\delta \in \Delta$ будем полагать $R(a, \delta)=R_{\delta}(a)=a \cdot \delta$.

ОпРЕДЕЛЕНИЕ 1.1. Допустим, что открытое покрытие $\mathscr{U}$ обладает свойствами

(1) существует ассоциированньй с $\mathscr{U}$ атлас $\mathscr{A}(\mathscr{U})$ расслоения $\xi$;

(2) $R_{\delta}(U)=U$ для всех $U \in \mathscr{U}$ и $\delta \in \Delta$.

Тогда $\mathscr{U}$ мы будем назьвать $(\xi, \Delta)$-покрытием.

ЗАмЕчАниЕ 1.1. Мы исходим из определения, согласно которому гладкое многообразие обладает счетной базой и потому паракомпактно. Кроме того, группа $\Delta$ конечна. Следовательно, для произвольного атласа $\mathscr{A}(\mathscr{V})$ расслоения $\xi$ по теореме 6.1 главы 3 книги [5] найдется вписанное в $\mathscr{V}$ локально конечное регулярное $\Delta$-покрытие $\mathscr{W}$. При этом множества вида $U=\cup_{\delta \in \Delta} W \cdot \delta, W \in \mathscr{W}$, образуют $(\xi, \Delta)$-покрытие $\mathscr{U}$ базы $B$.

Выберем карты $\xi_{U}, \xi_{V} \in \mathscr{A}(\mathscr{U})$, для которых $V \cap U \neq \varnothing$. Функция перехода $\xi_{V U}$ : $V \cap U \rightarrow T^{k}$ от $\xi_{U}$ к $\xi_{V}$ связывает их равенством

$$
\xi_{U}(a, t)=\xi_{V}\left(a, \xi_{V U}(a)+t\right)
$$

где $a \in V \cap U$ и $t \in T^{k}$. Определим отображение $\tau_{\delta}^{V U}: U \cap V \rightarrow T^{k}$ формулой

$$
\tau_{\delta}^{V U}=\xi_{V U} \circ R_{\delta}-\xi_{V U}
$$

ОПРЕДЕЛЕНИЕ 1.2. Пусть $d \tau_{\delta}^{V U} \equiv 0$ для всех $U, V \in \mathscr{U}$ и $\delta \in \Delta$. Тогда $\mathscr{A}(\mathscr{U})$ будет назьваться почти $\Delta$-атласом. Два таких атласа әквивалентны, если их объединение тоже является почти $\Delta$-атласом. Если $\mathscr{A}$ - класс эквивалентности почти $\Delta$-атласа $\mathscr{A}(\mathscr{U})$, то пару $\rho=(\xi, \mathscr{A})$ назовем почти $\Delta$-расслоением.

Рассмотрим главные расслоения $\xi$ и $\xi^{\prime}$ с проекциями $p: E \rightarrow B$ и $p^{\prime}: E^{\prime} \rightarrow B$ и структурной групшой $T^{k}$, а также гладкое отображение $f: E \rightarrow E^{\prime}$ со свойствами: $p=p^{\prime} \circ f$ и $f(v \cdot t)=f(v) \cdot t$ для всех $v \in E$ и $t \in T^{k}$. Тогда $f: \xi \rightarrow \xi^{\prime}$-морфизм над $B$. Допустим, что расслоения $\xi$ и $\xi^{\prime}$ обладают почти $\Delta$-структурами $\mathscr{A}$ и $\mathscr{A}^{\prime}$. Выберем атласы $\mathscr{A}(\mathscr{U}) \in \mathscr{A}, \mathscr{A}^{\prime}(\mathscr{U}) \in \mathscr{A}^{\prime}$ и множества $U, V \in \mathscr{U}, V \cap U \neq \varnothing$. Имеется функция перехода $\zeta_{V U}: V \cap U \rightarrow T^{k}$ от карты $\xi_{U}$ к карте $\xi_{V}^{\prime}$ при морфизме $f$. При этом для всех $a \in V \cap U$ и $t \in T^{k}$

$$
f \circ \xi_{U}(a, t)=\xi_{V}^{\prime}\left(a, \zeta_{V U}(a)+t\right) .
$$

Определим гладкое отображение $\sigma_{\delta}^{V U}: V \cap U \rightarrow T^{k}$, полагая

$$
\sigma_{\delta}^{V U}=\zeta_{V U} \circ R_{\delta}-\zeta_{V U}
$$


ОПрЕДЕЛЕНИЕ 1.3. Если $d \sigma_{\delta}^{V U} \equiv 0$ для всех $\xi_{U} \in \mathscr{A}(\mathscr{U}), \xi_{V}^{\prime} \in \mathscr{A}^{\prime}(\mathscr{U})$ и $\delta \in \Delta$, то $f$ назовем морфизмом почти $\Delta$-расслоений $\rho=(\xi, \mathscr{A})$ и $\rho^{\prime}=\left(\xi^{\prime}, \mathscr{A}^{\prime}\right)$.

Далее мы будем считать фиксированными многообразие $B$, группы $T^{k}, \Delta$ и действие $R: B \times \Delta \rightarrow B$, а также полагать $G=\Delta \times T^{k}$. Совокупность почти $\Delta$-расслоений над $B$ со структурной группой $T^{k}$ и их морфизмов образует категорию $\mathscr{K}\left(B, T^{k}, \Delta, R\right)$. Если $\rho^{\prime}=\left(\xi^{\prime}, \mathscr{A}^{\prime}\right)$ и $\rho^{\prime \prime}=\left(\xi^{\prime}, \mathscr{A}^{\prime \prime}\right)$ - объекты категории $\mathscr{K}\left(B, T^{k}, \Delta, R\right)$, то найдутся атласы $\mathscr{A}^{\prime}(\mathscr{U}) \in \mathscr{A}^{\prime}$ и $\mathscr{A}^{\prime \prime}(\mathcal{U}) \in \mathscr{A}^{\prime \prime}$, ассоциированные с одним и тем же покрытием $\mathscr{U}$ базы $B$ (см., например, [6, гл. 5, конец п. 2]). Пусть $\left\{\xi_{V U}^{\prime}\right\}$ и $\left\{\xi_{V U}^{\prime \prime}\right\}-$ наборы функций перехода атласов $\mathscr{A}^{\prime}(\mathscr{U})$ и $\mathscr{A}^{\prime \prime}(\mathscr{U})$ соответственно. Тогда суммы $\xi_{V U}=\xi_{V U}^{\prime}+\xi_{V U}^{\prime \prime}$ образуют коцикл и потому существует главное расслоение $\xi$ с базой $B$, структурной группой $T^{k}$ и атласом $\mathscr{A}(\mathscr{U})$, обладающим системой функций перехода $\left\{\xi_{V U}\right\}$ (см. [6, гл. 5, п. 3]). Очевидно, $\mathscr{A}(\mathscr{U})$ - почти $\Delta$-атлас. Если $\mathscr{A}$ - его класс эквивалентности, то пара $\rho=(\xi, \mathscr{A})$ является почти $\Delta$-расслоением. При этом формула $[\rho]=\left[\rho^{\prime}\right]+\left[\rho^{\prime \prime}\right]$ корректно определяет групповую операцию на множестве $\mathscr{B}\left(B, T^{k}, \Delta, R\right)$ классов эквивалентности объектов категории $\mathscr{K}\left(B, T^{k}, \Delta, R\right)$. Нейтральный элемент групшы $\mathscr{B}\left(B, T^{k}, \Delta, R\right)$ представляет собой класс эквивалентности пары $\rho_{0}=\left(\xi_{0}, \mathscr{A}_{0}\right)$, где $\xi_{0}$ - расслоение-произведение $B$ на $T^{k}$, а $\mathscr{A}_{0}$ содержит атлас $\left\{\operatorname{id}_{B \times T^{k}}\right\}$.

ЗАмЕчАниЕ 1.2. Отметим, что операция сложения на множестве классов эквивалентности главньг расслоений с фиксированной базой $B$ и структурной грушой $T^{1}$ двумя различными способами описана в [7]. Для ассоциированных векторных расслоений соответствующая операция рассматривается в разделе 10 книги [8].

ЗАмЕчАниЕ 1.3 . Элементы $(\xi, \Delta)$-покрытия $\mathscr{U}$ и их пересечения не обязаны быть связньми. Поэтому тождества $d \tau_{\delta}^{V U} \equiv 0$ и $d \sigma_{\delta}^{V U} \equiv 0$ равносильны тому, что отображения $\tau_{\delta}^{V U}$ и $\sigma_{\delta}^{V U}$ только локально постоянны. Добавим, что расслоение-произведение $\xi_{0}$ может обладать почти $\Delta$-структурой, отличной от $\mathscr{A}_{0}$.

2. Многозначные действия и $G$-связности. Пусть $\xi$-главное расслоение с проекцией $p: E \rightarrow B$ и структурной группой $T^{k}$. Каждая его карта $\xi_{U}: U \times T^{k} \rightarrow E_{U}$ позволяет определить действие $R^{U}: E_{U} \times \Delta \rightarrow E_{U}$ группы $\Delta$ на подмногообразии $E_{U}=p^{-1}(U) \subset E$ с помощью формулы

$$
R^{U}\left(\xi_{U}(a, t), \delta\right)=\xi_{U}(a \cdot \delta, t) .
$$

При этом для каждого $\delta \in \Delta$

$$
p \circ R_{\delta}^{U}=R_{\delta} \circ p .
$$

Если $\xi_{V}: V \times T^{k} \rightarrow E_{V}-$ другая карта расслоения $\xi$ и $V \cap U \neq \varnothing$, то действия $R^{U}$ и $R^{V}$ связаны на $E_{V \cap U}$ равенством

$$
R_{\delta}^{U}(v)=R_{\delta}^{V}(v) \cdot \tau_{\delta}^{V U}(p(v)),
$$

где отображение $\tau_{\delta}^{V U}: V \cap U \rightarrow T^{k}$ определено формулой (1.2).

ОПРЕДЕЛЕНИЕ 2.1. Пусть $\mathscr{A}(\mathscr{U})$ - атлас главного расслоения $\xi$, а отображения $R^{U}$ : $E_{U} \times \Delta \rightarrow E_{U}$ заданы с помошью карт $\xi_{U} \in \mathscr{A}(\mathscr{U})$ и формулы $(2.1)$. Набор $\mathscr{R}=\left\{R^{U}\right.$

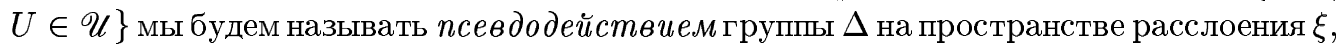
ассоциированным с $\mathscr{A}(\mathscr{U})$. Если $d \tau_{\delta}^{V U} \equiv 0$ для всех $U, V \in \mathscr{U}$ и $\delta \in \Delta$ (т.е. $\mathscr{A}(\mathscr{U})-$ почти $\Delta$-атлас), то $\mathscr{R}$ назовем многозначным действием. 
ЗАмЕчаниЕ 2.1. Из равенств (2.1) и (2.2) следует, что $R_{\delta}^{U}: E_{U} \rightarrow E_{U}$ - автоморфизм сужения над $U$ расслоения $\xi$. Поэтому для многозначного действия $\mathscr{R}$ набор $\{\mathscr{R} \delta$ | $U \in \mathscr{U}\}$ естественно назвать многозначным автоморфизмом расслоения $\xi$. При этом $\Delta$ становится группой многозначных автоморфизмов.

Рассмотрим главное расслоение $\xi^{\prime}$ с проекцией $p^{\prime}: E^{\prime} \rightarrow B$ и структурной группой $T^{k}$, морфизм $f: \xi \rightarrow \xi^{\prime}$ и $T^{k}$-связности $H$ на $E$ и $H^{\prime}$ на $E^{\prime}$, удовлетворяющие равенству $H_{f(v)}^{\prime}=d f\left(H_{v}\right)$ при всех $v \in E$. Выберем карты $\xi_{U}$ и $\xi_{V}^{\prime}$ расслоений $\xi$ и $\xi^{\prime}$ и элемент $\delta \in \Delta$. Предположим, что $V \cap U \neq \varnothing$.

ПРЕДЛОЖЕНИЕ 2.1. Если связность $Н$ инвариантна относительно преобразования $R_{\delta}^{U}: E_{U} \rightarrow E_{U}$, то $H^{\prime}$ инвариантна на $E_{V \cap U}^{\prime}$ относительно $R_{\delta}^{\prime V}: E_{V}^{\prime} \rightarrow E_{V}^{\prime}$ тогда и только тогда, когда определенное формулой (1.4) отображсние $\sigma_{\delta}^{V U}$ : $V \cap U \rightarrow T^{k}$ локально постоянно.

ДокАЗАТЕЛЬСтво. Предположим сначала, что связность $H^{\prime}$ инвариантна на $E_{V \cap U}^{\prime}$ относительно $R_{\delta}^{\prime V}$. Выберем компоненту связности $W$ пересечения $V \cap U$, точки $a_{0}$, $a_{1} \in W$ и путь $x: I \rightarrow W$ с началом $x(0)=a_{0}$ и конщом $x(1)=a_{1}$. Для некоторого $t_{0} \in T^{k}$ положим $v_{0}=\xi_{U}\left(a_{0}, t_{0}\right)$. Рассмотрим горизонтальньй относительно связности $H$ лифт $y$ пути $x$ с начальной точкой $y(0)=v_{0}$. Тогда найдется такое $t_{1} \in T^{k}$, что $y(1)=v_{1}=\xi_{U}\left(a_{1}, t_{1}\right)$. Положим $y_{U}=f \circ R_{\delta}^{U} \circ y$ и $y_{V}=R_{\delta}^{\prime} \circ f \circ y$. Пути $y_{U}: I \rightarrow E^{\prime}$ и $y_{V}: I \rightarrow E^{\prime}$ горизонтальны относительно связности $H^{\prime}$. Кроме того, $p^{\prime} \circ y_{U}=p^{\prime} \circ y_{V}$. Поэтому существует элемент $t \in T^{k}$, при всех $s \in I$ удовлетворяющий равенству $y_{U}(s)=y_{V}(s) \cdot t$. В частности,

$$
f\left(R_{\delta}^{U}\left(v_{i}\right)\right)=R_{\delta}^{\prime V}\left(f\left(v_{i}\right)\right) \cdot t
$$

для $i=0,1$. С другой стороны в силу (1.3) и (2.1)

$$
f \circ R_{\delta}^{U}\left(v_{i}\right)=R_{\delta}^{\prime V}\left(f\left(v_{i}\right)\right) \cdot \sigma_{\delta}^{V U}\left(a_{i}\right)
$$

Согласно (2.4) и (2.5) $\sigma_{\delta}^{V U}\left(a_{i}\right)=t$. Этим доказано, что $\sigma_{\delta}^{V U}$ локально постоянно.

Допустим далее, что для $i=0,1$ и любого $\delta \in \Delta$ верно предыдущее равенство. Положим $S_{t}\left(v^{\prime}\right)=v^{\prime} \cdot t$ для всех $v^{\prime} \in E^{\prime}$. В силу $(2.5) S_{-t} \circ f \circ R_{\delta}^{U}=R_{\delta}^{\prime V} \circ f$ на $E_{V \cap U}$. Рассмотрим формы $\omega$ и $\omega^{\prime}$ связностей $H$ и $H^{\prime}$. По определению $S_{-t}^{*} \omega^{\prime}=\omega^{\prime}$. Согласно условию $f^{*} \omega^{\prime}=\omega$ и $\left(R_{\delta}^{U}\right)^{*} \omega=\omega$. Поэтому $f^{*}\left(R_{\delta}^{\prime V}\right)^{*} \omega^{\prime}=\left(S_{-t} \circ f \circ R_{\delta}^{U}\right)^{*} \omega^{\prime}=\left(R_{\delta}^{U}\right)^{*} f^{*} S_{-t}^{*} \omega^{\prime}=$ $\left(R_{\delta}^{U}\right)^{*} f^{*} \omega^{\prime}=\left(R_{\delta}^{U}\right)^{*} \omega=\omega=f^{*} \omega^{\prime}$. Так как $f: E \rightarrow E^{\prime}-$ диффеоморфизм, то из доказанного следует равенство $\left(R_{\delta}^{\prime V}\right)^{*} \omega^{\prime}=\omega^{\prime}$.

Из предложения 2.1 вытекает ряд важных следствий. Для их компактной формулировки нам потребуются также новые определения.

СледСтВИЕ 1. Рассмотрим главное расслоение $\xi$ проекиией $p: E \rightarrow B u$ структурной группой $T^{k}$ и псевдодействие $\mathscr{R}$ группы $\Delta$ на $E$, ассоциированное с атласом $\mathscr{A}(\mathscr{U})$. На пространстве расслоения $\xi$ существует $T^{k}$-связность $H$, инвариантная относительно всех $R^{U} \in \mathscr{R}$, тогда и только тогда, когда $\mathscr{A}(\mathscr{U})-$ почти $\Delta$-атлас, а $\mathscr{R}$ - многозначное действие группы $\Delta$. 
ОПРЕДЕЛЕНИЕ 2.2. Если в обозначениях следствия 1 связность $H$ инвариантна относительно всех $R^{U} \in \mathscr{R}$, то мы будем говорить, что она инвариантна относительно многозначного действия $\mathscr{R}$ групшы $\Delta$ на $E$.

СлЕДСТвИЕ 2. Предположим, что $\rho=(\xi, \mathscr{A})$ - почти $\Delta$-расслоение $u T^{k}$-связность $H$ на пространстве $E$ главного расслоения $\xi$ инвариантна относительно многозначного действия группы $\Delta$, ассочиированного с атласом $\mathscr{A}(\mathscr{U}) \in \mathscr{A}$. Ecли $\mathscr{A}^{\prime}\left(\mathcal{U}^{\prime}\right)$ - другой атлас главного расслоения $\xi$, то связность Н инвариантна относительно ассоииированного с ним псевдодействия группь $\Delta$ на $E$ тогда $u$ только тогда, когда $\mathscr{A}^{\prime}\left(\mathscr{U}^{\prime}\right)$ - почти $\Delta$-атлас $и \mathscr{A}^{\prime}\left(\mathcal{U}^{\prime}\right) \in \mathscr{A}$.

ОПрЕдЕЛЕНИЕ 2.3. Пусть $G=\Delta \times T^{k}, \rho=(\xi, \mathscr{A})$ - почти $\Delta$-расслоение и $T^{k}$-связность $H$ на его пространстве $E$ инвариантна относительно многозначных действий группы $\Delta$, ассоциированных с атласами $\mathscr{A}(\mathscr{U}) \in \mathscr{A}$. Тогда $H$ будет назьваться $G$-связностью.

СлЕДСТВИЕ 3. Допустим, что $\rho=(\xi, \mathscr{A}) u \rho^{\prime}=\left(\xi^{\prime}, \mathscr{A}^{\prime}\right)-$ почти $\Delta$-расслоения, $\omega u \omega^{\prime}-\oint о р м и T^{k}$-связности $H u$ G-связности $H^{\prime}$ на пространствах $E$ u $E^{\prime}$ расслоений $\rho$ и $\rho^{\prime}, f: \xi \rightarrow \xi^{\prime}-$ морфизм главных $T^{k}-$ расслоений $u \omega=f^{*} \omega^{\prime}$. Тогда $H-G$-связность на $E$ в том и только в том случае, если $f: \rho \rightarrow \rho^{\prime}-$ морфизм почти $\Delta$-расслоений.

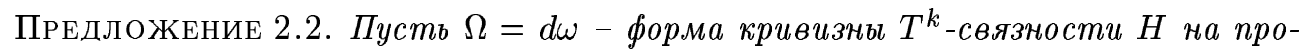
странстве Е главного расслоения $\xi, F$-замкнутая 2-форма на базе $B$ со значениями в $\mathbb{R}^{k}$, удовлетворяющая равенству $\Omega=p^{*} F$. Форма $F$ инвариантна относительно действия группы $\Delta$ на В в том и только в том случае, если $\xi$ обладает почти $\Delta$-структурой, относительно которой $H$ является $G$-связностью.

ДоКАЗАТЕЛЬСтво. Предположим, что $\mathscr{A}$ - почти $\Delta$-структура, относительно которой $H-G$-связность. Рассмотрим атлас $\mathscr{A}(\mathscr{U}) \in \mathscr{A}$ и множество $U \in \mathscr{U}$. Тогда $\left(R_{\delta}^{U}\right)^{*} \omega=\omega$ на $E_{U}$ для любого $\delta \in \Delta$. Отсюда и из (2.2) следует равенство $p^{*}\left(R_{\delta}^{*} F-F\right)=0$. Последнее равносильно инвариантности $F$ относительно $R_{\delta}$.

Пусть далее дано, что $R_{\delta}^{*} F=F$ для всех $\delta \in \Delta$. Выберем $(\xi, \Delta)$-покрытие $\mathscr{U}$ базы $B$, элементы которого односвязны. По определению существует ассоциированньй с $\mathscr{U}$ атлас $\overline{\mathscr{A}}(\mathscr{U})$ расслоения $\xi$.

Для произвольной карты $\bar{\xi}_{U} \in \overline{\mathscr{A}}(\mathscr{U})$ рассмотрим сечение $\bar{\sigma}_{U}: U \rightarrow E_{U}$, определенное формулой $\bar{\sigma}_{U}(a)=\bar{\xi}_{U}(a, 0)$, и локальную форму $\bar{\omega}_{U}=\bar{\sigma}_{U}^{*} \omega$ связности $H$. Положим $\omega_{U}^{\Delta}=(1 /|\Delta|) \sum_{\gamma \in \Delta} R_{\gamma}^{*} \bar{\omega}_{U}$. Тогда согласно допущению $d\left(\omega_{U}^{\Delta}-\bar{\omega}_{U}\right)=0$. В силу равенства $H_{1}(U)=0$ отсюда следует существование гладкого отображения $\mu_{U}: U \rightarrow \mathbb{R}^{k}$, для которого $d \mu_{U}=\omega_{U}^{\Delta}-\bar{\omega}_{U}$. Полагая

$$
\operatorname{Exp}(r)=\operatorname{Exp}\left(r^{1}, \ldots, r^{k}\right)=\left(r^{1}+\mathbb{Z}, \ldots, r^{k}+\mathbb{Z}\right)=r+\mathbb{Z}^{k}
$$

и $\xi_{U}(a, t)=\bar{\xi}_{U}\left(a, \operatorname{Exp}\left(\mu_{U}(a)\right)+t\right)$ для $a \in U$ и $t \in T^{k}$, определим отображения $\operatorname{Exp:~}$ $\mathbb{R}^{k} \rightarrow T^{k}$ и $\xi_{U}: U \times T^{k} \rightarrow E_{U}$. Набор $\mathscr{A}(\mathscr{U})=\left\{\xi_{U} \mid U \in \mathscr{U}\right\}$ представляет собой атлас главного расслоения $\xi$.

По построению $\sigma_{U}(a)=\xi_{U}(a, 0)=\bar{\sigma}_{U}(a) \cdot\left(\operatorname{Exp}\left(\mu_{U}(a)\right)\right)$ и $\omega_{U}=\sigma_{U}^{*} \omega=\bar{\omega}_{U}+d \mu_{U}=$ $\omega_{U}^{\Delta}$. Поэтому

$$
R_{\delta}^{*} \omega_{U}=\omega_{U}
$$


для любого $\delta \in \Delta$. Если $\mathscr{R}$ - псевдодействие группы $\Delta$ на $E$, ассоциированное с атласом $\mathscr{A}(\mathscr{U})$, то для любых $R^{U} \in \mathscr{R}$ и $\delta \in \Delta$ имеет место равенство

$$
R_{\delta}^{U} \circ \sigma_{U}=\sigma_{U} \circ R_{\delta}
$$

В силу (2.6) и (2.7)

$$
\left(\left(R_{\delta}^{U}\right)^{*} \omega-\omega\right) \circ d \sigma_{U}=0
$$

Пусть теперь $v \in E_{U}$ и $X \in T_{v} E$. Положим $X_{0}=d \sigma_{U}(d p(X))$ и $X_{1}=X-X_{0}$. Тогда $\left(\left(R_{\delta}^{U}\right)^{*} \omega-\omega\right)\left(X_{0}\right)=0$ согласно $(2.8)$, а $\left(\left(R_{\delta}^{U}\right)^{*} \omega-\omega\right)\left(X_{1}\right)=0$ в силу вертикальности вектора $X_{1}$. В результате: $\left(\left(R_{\delta}^{U}\right)^{*} \omega-\omega\right)(X)=0$ для всех $U \in \mathscr{U}, \delta \in \Delta, v \in E_{U}$ и $X \in T_{v} E$. Это значит, что форма $\omega$ инвариантна относительно псевдодействия $\mathscr{R}$. Но тогда $\mathscr{A}(\mathscr{U})$ - почти $\Delta$-атлас, $\mathscr{R}$ - ассоциированное с ним многозначное действие группы $\Delta$ на пространстве расслоения $\xi$, а $H-G$-связность.

Рассмотрим почти $\Delta$-расслоения $\rho=(\xi, \mathscr{A})$ и $\rho^{\prime}=\left(\xi^{\prime}, \mathscr{A}^{\prime}\right)$ с проекциями $p: E \rightarrow B$ и $p^{\prime}: E^{\prime} \rightarrow B$, а также формы $\omega$ и $\omega^{\prime} G$-связностей $H$ на $E$ и $H^{\prime}$ на $E^{\prime}$. Предположим, что существует морфизм главных расслоений $f: \xi \rightarrow \xi^{\prime}$. Тогда на базе $B$ найдется 1-форма $D$, удовлетворяющая равенству $f^{*} \omega^{\prime}-\omega=p^{*} D$.

ПРЕДЛОЖЕНИЕ 2.3. Отображение $f$ является морфизмом почти $\Delta$-расслоений $\rho$ и $\rho^{\prime}$ в том и только том случае, если форма $D$ инвариантна относительно действия $R$ әруппь $\Delta$.

ДокАЗАТЕЛЬСтво. По условию $\omega^{*}=f^{*} \omega^{\prime}-$ форма некоторой $T^{k}$-связности $H^{*}$ на $E$. По следствию 3 предложения $2.1 f: \rho \rightarrow \rho^{\prime}$ - морфизм почти $\Delta$-расслоений тогда и только тогда, когда $H^{*}-G$-связность. Последнее равносильно существованию такого атласа $\mathscr{A}(\mathscr{U}) \in \mathscr{A}$, что ассоциированное с ним многозначное действие $\mathscr{R}$ групшы $\Delta$ на $E$ удовлетворяет равенствам $\left(R_{\delta}^{U}\right)^{*} \omega^{*}=\omega^{*}$ при всех $U \in \mathscr{U}$ и $\delta \in \Delta$. Так как форма $\omega$ инвариантна относительно всех $R_{\delta}^{U}$, предыдущее верно в том и только в том случае, если $\left(R_{\delta}^{U}\right)^{*} p^{*} D=p^{*} D$. В силу (2.2) полученное равенство имеет место тогда и только тогда, когда $p^{*}\left(R_{\delta}^{*} D-D\right)=0$. Последнее эквивалентно инвариантности формы $D$ относительно $R_{\delta}$.

ПРЕДЛОЖЕНИЕ 2.4. Пусть $\rho=(\xi, \mathscr{A})$ - почти $\Delta$-расслоение с проекиией $p$ : $E \rightarrow B$. Тогда $[\rho]=0$ в $\mathscr{B}\left(B, T^{k}, \Delta, R\right)$ в том и только в том случае, если на $E$ существует $G$-связность с тривиальной группой голономии.

ДокАЗАтЕЛЬСтво. Допустим, что $H-G$-связность на $E$ с тривиальной группой голономии. Зафиксируем точки $v \in E$ и $b=p(v)$. Для произвольной точки $a \in B$ выберем кусочно-гладкий путь $x: I \rightarrow B$ с началом $x(0)=b$ и концом $x(1)=a$ и рассмотрим его горизонтальньй лифт $\bar{x}: I \rightarrow E$ с начальной точкой $\bar{x}(0)=v$. Положим $\sigma(a)=\bar{x}(1)$. Этим определено гладкое сечение $\sigma: B \rightarrow E$ расслоения $\xi$.

Пусть $\delta \in \Delta, \mathscr{A}(\mathscr{U}) \in \mathscr{A}, U \in \mathscr{U}$ и $K U-$ компонента связности множества $U$. Выберем произвольные точки $a_{0}, b_{0} \in K U$ и положим $a_{1}=a_{0} \cdot \delta, b_{1}=b_{0} \cdot \delta$. Еcли $x_{i}, y_{i}: I \rightarrow B$ - кусочно-гладкие пути с общим началом $b$ и концами $x_{i}(1)=a_{i}$, $y_{i}(1)=b_{i}, i=0,1$, a $\bar{x}_{i}, \bar{y}_{i}: I \rightarrow E-$ их горизонтальные лифты с начальной точкой $v$, то $\bar{x}_{i}(1)=\sigma\left(a_{i}\right), \bar{y}_{i}(1)=\sigma\left(b_{i}\right), i=0,1$. 
Рассмотрим кусочно-гладкий путь $z_{0}: I \rightarrow K U$ с началом $a_{0}$ и конщом $b_{0}$. Тогда путь $z_{1}=z_{0} \cdot \delta$ лежит в $U$ и идет из точки $a_{1}$ в точку $b_{1}$. Пусть $\bar{z}_{0}: I \rightarrow E$-горизонтальньй лифт пути $z_{0}$ с началом $\bar{z}_{0}(0)=\bar{x}_{0}(1)=\sigma\left(a_{0}\right)$. При этом $\bar{x}_{0}+\bar{z}_{0}-$ горизонтальный лифт пути $x_{0}+z_{0}$ и $\left(\bar{x}_{0}+\bar{z}_{0}\right)(0)=v$. Следовательно, $\bar{z}_{0}(1)=\left(\bar{x}_{0}+\bar{z}_{0}\right)(1)=\sigma\left(b_{0}\right)$.

Положим $\bar{z}_{\delta}=R_{\delta}^{U} \circ \bar{z}_{0}$. Так как $H-G$-связность и $p \circ R_{\delta}^{U} \circ \bar{z}_{0}=R_{\delta} \circ p \circ \bar{z}_{0}=z_{1}$, то $\bar{z}_{\delta}$ - горизонтальньй лифт пути $z_{1}$. Кроме того, найдется такой элемент $t_{K U} \in T^{k}$, что $\bar{z}_{\delta}(0) \cdot t_{K U}=\sigma\left(a_{1}\right)$. При этом путь $\bar{z}_{1}=\bar{z}_{\delta} \cdot t_{K U}$ также является горизонтальным лифтом пути $z_{1}$ и имеет начало $\bar{z}_{1}(0)=\bar{x}_{1}(1)=\sigma\left(a_{1}\right)$. Следовательно, $\bar{x}_{1}+\bar{z}_{1}-$ горизонтальньй лифт пути $x_{1}+z_{1}$ и потому $\bar{z}_{1}(1)=\left(\bar{x}_{1}+\bar{z}_{1}\right)(1)=\sigma\left(b_{1}\right)$.

По построению $\sigma\left(a_{1}\right)=\sigma\left(R_{\delta}\left(a_{0}\right)\right)$ и $\sigma\left(b_{1}\right)=\sigma\left(R_{\delta}\left(b_{0}\right)\right)$. Кроме того, $\sigma\left(a_{1}\right)=\bar{z}_{1}(0)=$ $\left(R_{\delta}^{U} \circ \bar{z}_{0}(0)\right) \cdot t_{K U}=\left(R_{\delta}^{U} \circ \bar{x}_{0}(1)\right) \cdot t_{K U}=\left(R_{\delta}^{U}\left(\sigma\left(a_{0}\right)\right) \cdot t_{K U}\right.$. Согласно доказанному $\sigma\left(b_{1}\right)=\bar{z}_{1}(1)=\left(R_{\delta}^{U} \circ \bar{z}_{0}(1)\right) \cdot t_{K U}=\left(R_{\delta}^{U} \circ \bar{y}_{0}(1)\right) \cdot t_{K U}=\left(R_{\delta}^{U}\left(\sigma\left(b_{0}\right)\right) \cdot t_{K U} \cdot\right.$ Таким образом, для всех $a \in K U$ верно равенство

$$
\left(\sigma \circ R_{\delta}\right)(a)=\left(R_{\delta}^{U} \circ \sigma\right)(a) \cdot t_{K U}
$$

Определим отображение $f: B \times T^{k} \rightarrow E$ формулой $f(a, t)=\sigma(a) \cdot t$. Тогда $f-$ морфизм главных расслоений $\xi_{0}$ и $\xi$. Покажем, что он является морфизмом почти $\Delta$-расслоений $\rho_{0}$ и $\rho$. Для этого выберем атлас $\mathscr{A}(\mathscr{U}) \in \mathscr{A}$, карту $\xi_{U} \in \mathscr{A}(\mathscr{U})$ и рассмотрим функцию перехода $\zeta_{U}: U \rightarrow T^{k}$ от карты $\mathrm{id}_{B \times T^{k}}$ расслоения $\xi_{0}$ к карте $\xi_{U}$. Для компоненты $K U$ множества $U$ и произвольных $a \in K U$ и $t \in T^{k}$ справедливы равенства $f(a, t)=\xi_{U}\left(a, \zeta_{U}(a)+t\right)$ и $f(a \cdot \delta, t)=\xi_{U}\left(a \cdot \delta, \zeta_{U}(a \cdot \delta)+t\right)$. Поэтому $\xi_{U}\left(a \cdot \delta, \zeta_{U}(a \cdot \delta)+t\right)=\sigma(a \cdot \delta) \cdot t$. Согласно $(2.9)$ и $(2.1) \sigma(a \cdot \delta) \cdot t=\left(R_{\delta}^{U} \circ \sigma\right)(a) \cdot\left(t_{K U}+t\right)=$ $R_{\delta}^{U} \circ \xi_{U}\left(a, \zeta_{U}(a)+t\right) \cdot t_{K U}=\xi_{U}\left(a \cdot \delta, \zeta_{U}(a)+t+t_{K U}\right)$. Из полученньх равенств следует, что $\zeta_{U}(a \cdot \delta)=\zeta_{U}(a)+t_{K U}$ для всех $a \in K U$. Последнее означает, что разность $\zeta_{U} \circ R_{\delta}-\zeta_{U}$ локально постоянна, а $f: \rho_{0} \rightarrow \rho$-морфизм почти $\Delta$-расслоений.

В обратную сторону утверждение предложения очевидно.

3. Характеристические классы. Если $\Phi$ - замкнутая $n$-форма на многообразии $B$ со значениями в $\mathbb{R}^{k}$, а $[\Phi]$ - ее когомологический класс, то формула $I_{[\Phi]}([c])=\int_{c} \Phi$ определяет гомоморфизм $I_{[\Phi]}: H_{n}(B) \rightarrow \mathbb{R}^{k}$.

Обозначим символом $\Lambda_{\Delta}^{n}\left(B, \mathbb{R}^{k}\right)$ группу инвариантных относительно действия группы $\Delta$ внешних $n$-форм на $B$ со значениями в $\mathbb{R}^{k}$, а символом $H_{\Delta}^{n}\left(B, \mathbb{R}^{k}\right)$ - группу гомологий коцепного комплекса

$$
\cdots \rightarrow \Lambda_{\Delta}^{n-1}\left(B, \mathbb{R}^{k}\right) \stackrel{d}{\rightarrow} \Lambda_{\Delta}^{n}\left(B, \mathbb{R}^{k}\right) \stackrel{d}{\rightarrow} \Lambda_{\Delta}^{n+1}\left(B, \mathbb{R}^{k}\right) \rightarrow \cdots
$$

Положим

$$
H_{\Delta}^{n}\left(B, \mathbb{R}^{k} \mid \mathbb{Z}^{k}\right)=\left\{[\Phi]_{\Delta} \in H_{\Delta}^{n}\left(B, \mathbb{R}^{k}\right) \mid \operatorname{im} I_{[\Phi]} \subset \mathbb{Z}^{k}\right\} .
$$

Пусть $\rho=(\xi, \mathscr{A})$-почти $\Delta$-расслоение с проекцией $p: E \rightarrow B$ и $\omega$ - форма $G$-связности $H$ на $E$. Тогда существует замкнутая 2-форма $F$ на $B$, удовлетворяющая равенству $d \omega=p^{*} F$. Согласно предложению $2.2 F \in \Lambda_{\Delta}^{2}\left(B, \mathbb{R}^{k}\right)$. Так как $\xi$-главное расслоение со структурной группой $T^{k}$, а $H-T^{k}$-связность, то im $I_{[F]} \subset \mathbb{Z}^{k}$.

Если $\rho^{\prime}=\left(\xi^{\prime}, \mathscr{A}^{\prime}\right)$ - другое почти $\Delta$-расслоение с проекцией $p^{\prime}: E^{\prime} \rightarrow B, \omega^{\prime}-$ форма $G$-связности $H^{\prime}$ на $E^{\prime}, F^{\prime}$ - замкнутая 2-форма на $B, d \omega^{\prime}=p^{*} F^{\prime}$ и $f: \rho \rightarrow \rho^{\prime}$-морфизм, 
то по предложению $2.3 f^{*} \omega^{\prime}=\omega+p^{*} D$, где $D \in \Lambda_{\Delta}^{1}\left(B, \mathbb{R}^{k}\right)$. При этом $\left[F^{\prime}\right]_{\Delta}=[F]_{\Delta}$. Таким образом, когомологический класс $[F]_{\Delta}$ является инвариантом расслоения $\rho$ в категории $\mathscr{K}\left(B, T^{k}, \Delta, R\right)$. Как обычно, он будет называться характеристическим классом почти $\Delta$-расслоения $\rho=(\xi, \mathscr{A})$. При этом формула $\eta([\rho])=[F]_{\Delta}$ определяет гомоморфизм $\eta: \mathscr{B}\left(B, T^{k}, \Delta, R\right) \rightarrow H_{\Delta}^{2}\left(B, \mathbb{R}^{k} \mid \mathbb{Z}^{k}\right)$.

ПРЕДЛОЖЕНИЕ 3.1. Для гомоморфизма $\eta$ существует правый обратный гомоморфизм $\bar{\eta}: H_{\Delta}^{2}\left(B, \mathbb{R}^{k} \mid \mathbb{Z}^{k}\right) \rightarrow \mathscr{B}\left(B, T^{k}, \Delta, R\right)$.

ДокАЗАТЕЛЬСТво. Пусть $F$ - замкнутая и инвариантная относительно действия группы $\Delta$ 2-форма на $B$ и

$$
[F]_{\Delta} \in H_{\Delta}^{2}\left(B, \mathbb{R}^{k} \mid \mathbb{Z}^{k}\right) .
$$

Для многообразия $B$ найдется (см., например, [5, гл. III, п. 6]) открытое $\Delta$-покрытие $\mathscr{U}$, обладающее свойствами

(1) $H_{1}(U)=0$ и $H_{2}(U)=0$ для всех $U \in \mathscr{U}$;

(2) $H_{1}(V \cap U)=0$ для любых $U, V \in \mathscr{U}$.

Зафиксируем точку $b \in B$. Для любого $U \in \mathscr{U}$ выберем 1-форму $\omega_{U}$ на $U$, удовлетворяющую равенству $d \omega_{U}=\left.F\right|_{U}$. В каждой компоненте связности $K U$ множества $U$ зафиксируем точку $a_{K U}$. Символом $x_{K U}: I \rightarrow B$ обозначим некоторьй путь с началом $x_{K U}(0)=b$ и конщом $x_{K U}(1)=a_{K U}$. Для произвольной точки $a \in K U$ зафиксируем путь $y_{K U}^{a}: I \rightarrow K U$ с началом $y_{K U}^{a}(0)=a_{K U}$ и конщом $y_{K U}^{a}(1)=a$.

Рассмотрим далее компоненту связности $K(V U)$ пересечения $V \cap U \neq \varnothing$ для некоторых $U, V \in \mathscr{U}$. Найдутся такие компоненты связности $K U$ и $K V$ множеств $U$ и $V$, что $K(V U) \subset K V \cap K U$. Поэтому для любой точки $a \in K(V U)$ определена петля

$$
x_{K(V U)}^{a}=x_{K V}+y_{K V}^{a}-y_{K U}^{a}-x_{K U}
$$

Пусть $\mathscr{Z}_{n}(B)$ и $\mathscr{B}_{n}(B)$ - группы $n$-мерных сингулярных циклов и границ многообразия $B$ с цельми коэффициентами. Если $x \in \mathscr{B}_{1}(B)$, то имеется сингулярная 2 -цепь $c_{x} \mathrm{c}$ гранищей $\partial c_{x}=x$. Положим

$$
h_{0}(x)=\operatorname{Exp}\left(\int_{c_{x}} F\right)
$$

где $\operatorname{Exp:~} \mathbb{R}^{k} \rightarrow T^{k}=\mathbb{R}^{k} / \mathbb{Z}^{k}$ - фактор-отображение, определенное ранее в доказательстве предложения 2.2. Если $c_{x}^{\prime}$ - другая 2 -цеп с той же границей $\partial c_{x}^{\prime}=x$, то $c_{x}^{\prime}-c_{x} \in \mathscr{Z}_{2}(B)$. В силу (3.1) $\operatorname{Exp}\left(\int_{c_{x}^{\prime}-c_{x}} F\right)=0$, где нуль в правой части обозначает нейтральный элемент адлитивной групшы $T^{k}$. Отсюда следует, что формула $(3.2)$ корректно определяет гомоморфизм $h_{0}: \mathscr{B}_{1}(B) \rightarrow T^{k}$. Так как $\operatorname{Ext}\left(H_{1}(B), T^{k}\right)=0$, то для него существует продолжение $h: \mathscr{Z}_{1}(B) \rightarrow T^{k}$.

Полагая для каждой компоненты $K(V U)$ множества $V \cap U$ и произвольной точки $a \in K(V U)$

$$
\xi_{V U}(a)=h\left(x_{K(V U)}^{a}\right)-\operatorname{Exp}\left(\int_{y_{K V}^{a}} \omega_{V}\right)+\operatorname{Exp}\left(\int_{y_{K U}^{a}} \omega_{U}\right)
$$

мы определим отображение $\xi_{V U}: V \cap U \rightarrow T^{k}$. 
Зафиксируем точку $a_{K(V U)} \in K(V U)$ и рассмотрим путь $z: I \rightarrow K(V U)$ с началом $z(0)=a_{K(V U)}$ и конщом $z(1)=a$. Положим $y_{K U}=y_{K U}^{a^{\prime}}, y_{K V}=y_{K V}^{a^{\prime}}$ и $x_{K(V U)}=$ $x_{K(V U)}^{a^{\prime}}$ при $a^{\prime}=a_{K(V U)}$. Пусть также

$$
z_{K U}^{a}=x_{K U}+y_{K U}+z-y_{K U}^{a}-x_{K U}, \quad z_{K V}^{a}=x_{K V}+y_{K V}+z-y_{K V}^{a}-x_{K V} .
$$

Тогда $x_{K(V U)}^{a}=-z_{K V}^{a}+x_{K(V U)}+z_{K U}^{a}$ и потому

$$
h\left(x_{K(V U)}^{a}\right)=-h\left(z_{K V}^{a}\right)+h\left(x_{K(V U)}\right)+h\left(z_{K U}^{a}\right) .
$$

По свойству (1) покрытия $\mathscr{U}$ циклы $z_{K U}^{a}$ и $z_{K V}^{a}$ являются границами. Следовательно, $h\left(z_{K U}^{a}\right)=h_{0}\left(z_{K U}^{a}\right)$ и $h\left(z_{K V}^{a}\right)=h_{0}\left(z_{K V}^{a}\right)$. При этом

$$
h\left(x_{K(V U)}^{a}\right)-h\left(x_{K(V U)}\right)=\operatorname{Exp}\left(\int_{y_{K U}+z-y_{K U}^{a}} \omega_{U}-\int_{y_{K V}+z-y_{K V}^{a}} \omega_{V}\right) .
$$

Отсюда и из (3.3) получим

$$
\xi_{V U}(a)-\xi_{V U}\left(a_{K(V U)}\right)=\operatorname{Exp}\left(\int_{z}\left(\omega_{U}-\omega_{V}\right)\right)
$$

Это значит, что отображение $\xi_{V U}: V \cap U \rightarrow T^{k}$ дифференцируемо и

$$
\theta \circ d \xi_{V U}=\omega_{U}-\omega_{V}
$$

где $\theta$ - каноническая 1-форма на группе $T^{k}$ со значениями в ее алгебре Ли $\mathbb{R}^{k}$.

Рассмотрим произвольньй элемент $\delta \in \Delta$. Так как $\mathscr{U}-\Delta$-покрытие, то множество $K^{\prime}(V U)=R_{\delta}(K(V U))$ также представляет собой компоненту связности пересечения $V \cap U$. Выберем пути $z_{1}: I \rightarrow K^{\prime}(V U)$ и $z_{2}: I \rightarrow K^{\prime}(V U)$ с общим началом $z_{1}(0)=z_{2}(0)=a_{K^{\prime}(V U)}$ и конщами $z_{1}(1)=a \cdot \delta$ и $z_{2}(1)=a_{K(V U)} \cdot \delta$. Тогда согласHо (3.4)

$$
\begin{array}{r}
\xi_{V U}(a \cdot \delta)-\xi_{V U}\left(a_{K^{\prime}(V U)}\right)=\operatorname{Exp}\left(\int_{z_{1}}\left(\omega_{U}-\omega_{V}\right)\right) \\
\xi_{V U}\left(a_{K(V U)} \cdot \delta\right)-\xi_{V U}\left(a_{K^{\prime}(V U)}\right)=\operatorname{Exp}\left(\int_{z_{2}}\left(\omega_{U}-\omega_{V}\right)\right)
\end{array}
$$

Вычитая из первого равенства второе, получим

$$
\xi_{V U}(a \cdot \delta)-\xi_{V U}\left(a_{K(V U)} \cdot \delta\right)=\operatorname{Exp}\left(\int_{-z_{2}+z_{1}}\left(\omega_{U}-\omega_{V}\right)\right) .
$$

Путь $-z_{2}+z_{1}$ лежит в $K^{\prime}(V U)$ и идет из точки $a_{K(V U)} \cdot \delta$ в точку $a \cdot \delta$. Точно такими же свойствами обладает и путь $z^{\prime}=R_{\delta} \circ z$. Так как $d\left(\omega_{U}-\omega_{V}\right)=0, H_{1}\left(K^{\prime}(V U)\right)=0$, $R_{\delta}^{*} \omega_{U}=\omega_{U}$ и $R_{\delta}^{*} \omega_{V}=\omega_{V}$, то

$$
\int_{-z_{2}+z_{1}}\left(\omega_{U}-\omega_{V}\right)=\int_{z^{\prime}}\left(\omega_{U}-\omega_{V}\right)=\int_{z}\left(\omega_{U}-\omega_{V}\right)
$$


Отсюда, из (3.6) и (3.4) следует, что

$$
\xi_{V U}(a \cdot \delta)-\xi_{V U}(a)=\xi_{V U}\left(a_{K(V U)} \cdot \delta\right)-\xi_{V U}\left(a_{K(V U)}\right)
$$

Если $U, V, W$ - элементы покрытия $\mathscr{U}$ и $K(W V U)$ - компонента связности пересечения $W \cap V \cap U$, то найдутся такие компоненты $K U, K V$ и $K W$ множеств $U, V$ и $W$, что $K(W V U) \subset K(W) \cap K(V) \cap K(U)$. При этом согласно (3.3) для произвольной точки $a \in K(W V U)$ имеет место равенство

$$
\xi_{U W}(a)+\xi_{W V}(a)+\xi_{V U}(a)=0 .
$$

Из (3.8) следует существование главного расслоения $\xi$ с базой $B$ и структурной группой $T^{k}$, обладающего атласом $\mathscr{A}(\mathscr{U})$, ассоциированньм с покрытием $\mathscr{U}$ и имеющим функции перехода $\left\{\xi_{V U} \mid V, U \in \mathscr{U}\right\}$. В силу (3.7) отображения $\tau_{V U}^{\delta}=\xi_{V U} \circ R_{\delta}-\xi_{V U}$ из $V \cap U$ в $T^{k}$ локально постоянны для всех $V, U \in \mathscr{U}, V \cap U \neq \emptyset$ и $\delta \in \Delta$. Поэтому $\mathscr{A}(\mathscr{U})$ - почти $\Delta$-атлас. Пусть $\mathscr{A}$ - класс эквивалентности атласа $\mathscr{A}(\mathscr{U})$. Положим $\rho=(\xi, \mathscr{A})$ и

$$
\bar{\eta}\left([F]_{\Delta}\right)=[\rho]
$$

Заменив в предыдущих построениях форму $F$ формой $\widetilde{F}=F+d A$, где $A$ - инвариантная относительно $\Delta$ 1-форма на $B$, получим гладкие отображения $\tilde{\xi}_{V U}: V \cap U \rightarrow T^{k}$, удовлетворяющие равенству

$$
\tilde{\xi}_{V U}(a)-\xi_{V U}(a)=\operatorname{Exp}\left(\int_{x_{K V}} A\right)-\operatorname{Exp}\left(\int_{x_{K U}} A\right) .
$$

При этом соответствующее набору $\left\{\tilde{\xi}_{V U} \mid U, V \in \mathscr{U}\right\}$ почти $\Delta$-расслоение $\tilde{\rho}$ будет изоморфно расслоению $\rho$. Следовательно, формула (3.9) корректно определяет отображение $\bar{\eta}: H^{2}\left(B, \mathbb{R}^{k} \mid \mathbb{Z}^{k}\right) \rightarrow \mathscr{B}\left(B, T^{k}, \Delta, R\right)$.

В силу (3.5) на пространстве расслоения $\rho$ существует $G$-связность $H$ с набором локальных форм связности $\left\{\omega_{U} \mid U \in \mathscr{U}\right\}$. По построению $\left.F\right|_{U}=d \omega_{U}$ - проекция на базу $B$ формы кривизны $d \omega$ связности $H$. Таким образом, $\eta[\rho]=[F]_{\Delta}$ и $\eta \circ \bar{\eta}=\mathrm{id}$.

Если все построения осуществить для форм $F, F^{\prime}$ и $F^{\prime \prime}=F+F^{\prime}$, где $[F],\left[F^{\prime}\right] \in$ $H^{2}\left(B, \mathbb{R}^{k} \mid \mathbb{Z}^{k}\right)$, то получатся почти $\Delta$-расслоения $\rho, \rho^{\prime}$ и $\rho^{\prime \prime}$, имеющие почти $\Delta$-атласы $\mathscr{A}(\mathscr{U}), \mathscr{A}^{\prime}(\mathscr{U})$ и $\mathscr{A}^{\prime \prime}(\mathscr{U})$ с функциями перехода $\left\{\xi_{V U}\right\},\left\{\xi_{V U}^{\prime}\right\}$ и $\left\{\xi_{V U}^{\prime \prime}=\xi_{V U}+\xi_{V U}^{\prime}\right\}$. Это значит, что $[\rho]+\left[\rho^{\prime}\right]=\left[\rho^{\prime \prime}\right]$, а $\bar{\eta}-$ гомоморфизм.

4. Почти $\Delta$-расслоения с нулевым характеристическим классом. Пусть $\rho=(\xi, \mathscr{A})$ - почти $\Delta$-расслоение с характеристическим классом $[\bar{F}]_{\Delta}=0$. Рассмотрим его проекцию $p: E \rightarrow B$ и $G$-связность $\bar{H}$, для которой форма связности $\bar{\omega}$ удовлетворяет равенству $d \bar{\omega}=p^{*} \bar{F}$. Тогда $\bar{F}=d A$, где $A-$ инвариантная относительно $\Delta$-форма на $B$ со значениями в пространстве $\mathbb{R}^{k}$. При этом согласно предложению $2.3 \omega=\bar{\omega}-p^{*} A-$ форма $G$-связности на $E$ и $d \omega=0$. Таким образом, для почти $\Delta$-расслоения $\rho$ включение $[\rho] \in \operatorname{ker} \eta$ эквивалентно существованию на его пространстве плоской $G$-связности $H$. 
Предположим, что $x: I \rightarrow B$ - кусочно-гладкая петля, а $\bar{x}: I \rightarrow E$ ее горизонтальный лифрт относительно плоской $G$-связности $H$. Тогда найдется элемент $\tau_{H}(x) \in T^{k}$, удовлетворяющий равенству $\bar{x}(1)=\bar{x}(0) \cdot \tau_{H}(x)$. Согласно [4] элемент $\tau_{H}(x)$ зависит только от гомологического класса цикла $x \in \mathscr{Z}_{1}(B)$. Кроме того, $\tau_{H}(x+y)=\tau_{H}(x)+\tau_{H}(y)$ и $\tau_{H}(-x)=-\tau_{H}(x)$. Следовательно, формула $\tau_{H}([x])=\tau_{H}(x)$ корректно определяет гомоморфизм $\tau_{H}: H_{1}(B) \rightarrow T^{k}$, которьй мы называем гомоморфизмом голономии связности $H$.

ПРЕДЛОЖЕНИЕ 4.1. Пусть $\rho=(\xi, \mathscr{A})$ - почти $\Delta$-расслоение с проекиией $p$ : $E \rightarrow B, H$ и $H^{*}$ - плоские $G$-связности на $E, \omega u \omega^{*}-$ их формы связности. Тогда $\omega^{*}=\omega+p^{*} A$, əде $A \in \mathscr{Z}_{\Delta}^{1}\left(B, \mathbb{R}^{k}\right), u \tau_{H^{*}}=\tau_{H}-\operatorname{Exp} \circ I_{[A]}$.

ДокАЗАтЕльство. Существование и инвариантность формы $A$ относительно $\Delta$ следуют из предложения 2.3 , ее замкнутость - из равенств $d \omega=d \omega^{*}=0$.

Рассмотрим петлю $x: I \rightarrow B$ и ее горизонтальные лифты $\bar{x}: I \rightarrow E$ и $\bar{x}^{*}: I \rightarrow E$ относительно связностей $H$ и $H^{*}$. Тогда найдется такой кусочно-гладкий путь $r: I \rightarrow \mathbb{R}^{k}$, что $\bar{x}^{*}=\bar{x} \cdot(\operatorname{Exp} \circ r)$. При этом

$$
\omega^{*}\left(\frac{d \bar{x}^{*}}{d s}\right)=\omega\left(\frac{d \bar{x}}{d s}\right)+\frac{d r}{d s}+A\left(\frac{d x}{d s}\right)
$$

Из полученного равенства следует, что $d r / d s=-A(d x / d s)$ или

$$
r(1)-r(0)=-\int_{x} A .
$$

По построению пути $r$ и гомоморфизма $\tau_{H}$ имеем

$$
\begin{aligned}
\bar{x}^{*}(1) & =\bar{x}(1) \cdot\left(\operatorname{Exp}(r(1))=\bar{x}(0) \cdot\left(\tau_{H}([x])+\operatorname{Exp}(r(1))\right.\right. \\
& =\bar{x}^{*}(0) \cdot\left(\operatorname{Exp}(-r(0))+\tau_{H}([x])+\operatorname{Exp}(r(1)) .\right.
\end{aligned}
$$

С другой стороны, $\bar{x}^{*}(1)=\bar{x}^{*}(0) \cdot\left(\tau_{H} *([x])\right.$. Таким образом, $\tau_{H}([x])+\operatorname{Exp}(r(1)-r(0))=$ $\tau_{H^{*}}([x])$. В силу (4.1) последнее влечет за собой доказываемое равенство.

Пусть для произвольного $n \in \mathbb{N}$

$$
\operatorname{Hom}^{\Delta}\left(H_{n}(B), \mathbb{R}^{k}\right)=\left\{h \in \operatorname{Hom}\left(H_{n}(B), \mathbb{R}^{k}\right) \mid h([x \cdot \delta])=h([x]) \forall \delta \in \Delta\right\}
$$

Для $[F]_{\Delta} \in H_{\Delta}^{n}\left(B, \mathbb{R}^{k}\right),[F] \in H^{2}\left(B, \mathbb{R}^{k}\right)$ и $[c] \in H_{n}(B)$ положим

$$
I_{\Delta}\left([F]_{\Delta}\right)=I_{[F]}
$$

Лемма. Отображсение $I_{\Delta}: H_{\Delta}^{n}\left(B, \mathbb{R}^{k}\right) \rightarrow \operatorname{Hom}^{\Delta}\left(H_{n}(B), \mathbb{R}^{k}\right)$, определенное формулой (4.2), является изоморфизмом. 
ДОкАЗАТЕЛЬСтво. Корректность определения $I_{\Delta}$ и его гомоморфность очевидны.

Если $I_{\Delta}\left([F]_{\Delta}\right)=0$, то согласно теореме Де Рама $F=d A$, где $A \in \Lambda^{n-1}\left(B, \mathbb{R}^{k}\right)$. Полагая $\widehat{A}=(1 /|\Delta|) \sum_{\delta \in \Delta} R_{\delta}^{*} A$, получим форму $\widehat{A} \in \Lambda_{\Delta}^{n-1}\left(B, \mathbb{R}^{k}\right)$. Легко видеть, что $d \widehat{A}=F$. Следовательно, $[F]_{\Delta}=0$ в $H_{\Delta}^{n}\left(B, \mathbb{R}^{k}\right)$ и $\operatorname{ker} I_{\Delta}=0$.

Пусть далее $h \in \operatorname{Hom}^{\Delta}\left(H_{n}(B), \mathbb{R}^{k}\right)$. По той же теореме Де Рама гомоморфизму $h$ coответствует форма $\Phi \in \mathscr{Z}^{n}\left(B, \mathbb{R}^{k}\right)$, удовлетворяющая равенству $\int_{c} \Phi=h([c])$ для всех $[c] \in H_{n}(B)$. Положим, как и выше, $F=(1 /|\Delta|) \sum_{\delta \in \Delta} R_{\delta}^{*} \Phi$. Тогда $F \in \mathscr{Z}_{\Delta}^{n}\left(B, \mathbb{R}^{k}\right)$ и

$$
\int_{c} F=\frac{1}{|\Delta|} \sum_{\delta \in \Delta} \int_{c \cdot \delta} \Phi=\frac{1}{|\Delta|} \sum_{\delta \in \Delta} h([c \cdot \delta])=h([c]) .
$$

Поэтому $I_{\Delta}\left([F]_{\Delta}\right)=h$ и гомоморфизм $I_{\Delta}$ сюръективен.

Для почти $\Delta$-расслоения $\rho$ с нулевым характеристическим классом и плоской $G$-связности $H$ на его пространстве рассмотрим гомоморфизм голономии $\tau_{H} \in \operatorname{Hom}\left(H_{1}(B)\right.$, $\left.T^{k}\right)$ и гомоморфизм $\operatorname{Exp}_{*}^{\Delta}: \operatorname{Hom}^{\Delta}\left(H_{1}(B), \mathbb{R}^{k}\right) \rightarrow \operatorname{Hom}\left(H_{1}(B), T^{k}\right)$, определенньй формулой $\operatorname{Exp}_{*}^{\Delta}(h)=\operatorname{Exp} \circ h$. Положим

$$
\eta_{0}([\rho])=\tau_{H}+\operatorname{im} \operatorname{Exp}_{*}^{\Delta} .
$$

ПРЕДЛОЖЕНИЕ 4.2. Формула (4.3) корректно определяет отображение

$$
\eta_{0}: \operatorname{ker} \eta \rightarrow \operatorname{Hom}\left(H_{1}(B), T^{k}\right) / \operatorname{im}_{\operatorname{Exp}_{*}^{\Delta}}^{\Delta} .
$$

ДокАЗАТЕЛЬСТво. Пусть $\rho$ и $\rho^{\prime}$ - почти $\Delta$-расслоения, $H$ и $H^{\prime}$ - плоские $G$-связности на их пространствах, $\omega$ и $\omega^{\prime}$ - формы связностей $H$ и $H^{\prime}$. Если $f: \rho \rightarrow \rho^{\prime}-$ морфизм, то $\omega^{*}=f^{*} \omega^{\prime}$ - форма некоторой плоской $G$-связности $H^{*}$ на пространстве расслоения $\rho$. При этом $\tau_{H^{*}}=\tau_{H^{\prime}}$ и справедливы все утверждения предложения 4.1. В такой ситуации для любых $[x] \in H_{1}(B)$ и $\delta \in \Delta$

$$
I_{[A]}([x \cdot \delta])=\int_{x \cdot \delta} A=\int_{x} R_{\delta}^{*} A=\int_{x} A=I_{[A]}([x]) .
$$

Следовательно, $\operatorname{Exp} \circ I_{[A]} \in \operatorname{im} \operatorname{Exp}_{*}^{\Delta}$ и потому смежные классы $\tau_{H}+\operatorname{im} \operatorname{Exp}_{*}^{\Delta}$ и $\tau_{H^{\prime}}+$ $\operatorname{im} \operatorname{Exp}_{*}^{\Delta}$ совпадают.

ПРеДлоЖенИЕ 4.3. Рассмотрим почти $\Delta$-расслоение $\rho=(\xi, \mathscr{A})$ с проекиией $p: E \rightarrow B$, плоскую $G$-связность $H$ на его пространстве $E$ и гомоморфизм голономии $\tau_{H}: H_{1}(B) \rightarrow T^{k}$. Тогда существует почти $\Delta$-атлас $\mathscr{A}(\mathscr{U}) \in \mathscr{A}$, обладающий свойствами

(1) локальные формы $\omega_{U}$ связности $H$ тождественно равны нулю для всех $U \in \mathscr{U}$

(2) функиии перехода $\xi_{V U}$ локально постоянны для любых $U, V \in \mathscr{U}, V \cap U \neq \varnothing$. При этом если $x: I \rightarrow B-$ петля, $0=s_{0}<s_{1}<\cdots<s_{l}=1$ - разбиение отрезка $I=[0,1], K U_{1}, \ldots, K U_{l}-$ компоненты связности әлементов покрытия $\mathscr{U}$, удовлетворяющ, ие условиям $x\left(\left[s_{i-1}, s_{i}\right]\right) \subset K U_{i}, u a_{i}=x\left(s_{i}\right)$, mo

$$
\tau_{H}([x])=\xi_{U_{1} U_{l}}\left(a_{l}\right)+\xi_{U_{l} U_{l-1}}\left(a_{l-1}\right)+\cdots+\xi_{U_{2} U_{1}}\left(a_{1}\right) .
$$


ДокАЗАтЕЛЬСтво. Рассмотрим покрытие $\mathscr{U}$ базы $B$ с теми же свойствами, что и в предложении 3.1. Пусть $\widehat{\mathscr{A}}(\mathscr{U})$ - ассоциированный с $\mathscr{U}$ атлас из $\mathscr{A},\left\{\hat{\xi}_{V U}\right\}-$ ее система функций перехода, а $\left\{\widehat{\omega}_{U}\right\}$ - соответствующий набор локальных форм связности $H$. По условию на связность и по выбору покрытия для всех $U \in \mathscr{U}$ найдутся функции $\hat{\phi}_{U}: U \rightarrow \mathbb{R}^{k}$, удовлетворяющие равенству $d \hat{\phi}_{U}=\widehat{\omega}_{U}$. Положим $\phi_{U}=\operatorname{Exp} \circ \hat{\phi}_{U}$ и

$$
\xi_{U}(a, t)=\hat{\xi}_{U}(a, t) \cdot\left(-\phi_{U}(a)\right)
$$

для карт $\hat{\xi}_{U} \in \hat{\mathscr{A}}(\mathscr{U})$, точек $a \in U$ и элементов $t \in T^{k}$. Этим определены карты $\xi_{U}: U \times T^{k} \rightarrow E_{U}$ расслоения $\xi$.

Если $U, V \in \mathscr{U}$ и $V \cap U \neq \varnothing$, то $\xi_{V U}=\phi_{V}+\hat{\xi}_{V U}-\phi_{U}-$ функция перехода от карты $\xi_{U}$ к карте $\xi_{V}$. Согласно (2.6) формы $\widehat{\omega}_{U} \circ d R_{\delta}-\widehat{\omega}_{U}$ тождественно равны нулю. Следовательно, $\phi_{U} \circ R_{\delta}-\phi_{U}-$ локально постоянное отображение для всех $U \in \mathscr{U}$ и $\delta \in \Delta$. А это значит, что $\mathscr{A}(\mathscr{U})=\left\{\xi_{U} \mid U \in \mathscr{U}\right\}-$ почти $\Delta$-атлас и $\mathscr{A}(\mathscr{U}) \in \mathscr{A}$.

Рассмотрим локальные формы $\omega_{U}$ связности $H$ относительно атласа $\mathscr{A}(\mathscr{U})$. В силу (4.5) $\widehat{\omega}_{U}=\omega_{U}+d \hat{\phi}_{U}$. По построению отображения $\hat{\phi}_{U}$ отсюда следует, что $\omega_{U} \equiv 0$ для всех $U \in \mathscr{U}$.

Для любых $U, V \in \mathscr{U}, V \cap U \neq \varnothing$, в силу (3.5) и согласно доказанному $d \xi_{V U} \equiv 0$. Поэтому функции перехода атласа $\mathscr{A}(\mathscr{U})$ локально постоянны.

Пусть $\bar{x}: I \rightarrow E$ - горизонтальньй лифт петли $x$ относительно $H$. В силу равенства нулю локальных форм связности существуют такие элементы $t_{i} \in T^{k}$, что

$$
\bar{x}(s)=\xi_{U_{i}}\left(x(s), t_{i}\right)
$$

для всех $s \in\left[s_{i-1}, s_{i}\right]$ и $i=1, \ldots, l$. Так как $a_{i} \in K U_{i-1} \cap K U_{i}$, из (4.6) следуют равенства $\bar{x}\left(s_{i}\right)=\xi_{U_{i-1}}\left(a_{i}, t_{i-1}\right)=\xi_{U_{i}}\left(a_{i}, t_{i}\right)$. Поэтому $t_{i}=\xi_{U_{i} U_{i-1}}\left(a_{i}\right)+t_{i-1}$. Применяя эту рекуррентную формулу и (4.6), получим

$$
\bar{x}(1)=\bar{x}(0) \cdot\left(\xi_{U_{1} U_{l}}\left(a_{l}\right)+\xi_{U_{l} U_{l-1}}\left(a_{l-1}\right)+\cdots+\xi_{U_{2} U_{1}}\left(a_{1}\right)\right) .
$$

Последнее равносильно (4.4).

\section{ПРЕДЛОЖЕНИЕ 4.4. Отображение $\eta_{0}$ является гомоморфизмом групп.}

ДокАЗАтЕльСтво. Рассмотрим почти $\Delta$-расслоения $\rho$ и $\rho^{\prime}$, для которьх $[\rho],\left[\rho^{\prime}\right] \in$ $\operatorname{ker} \eta$, и плоские $G$-связности $H$ и $H^{\prime}$ на их пространствах. Для некоторого покрытия $\mathscr{U}$ базы $B$ найдутся почти $\Delta$-атласы $\mathscr{A}(\mathscr{U})$ и $\mathscr{A}^{\prime}(\mathscr{U})$ этих расслоений, обладающие свойствами из предложения 4.3. Пусть $\left\{\xi_{V U}\right\}$ и $\left\{\xi_{V U}^{\prime}\right\}$ - системы функций перехода атласов $\mathscr{A}(\mathscr{U})$ и $\mathscr{A}^{\prime}(\mathscr{U})$. Согласно определению сложения в групше $\mathscr{B}\left(B, T^{k}, \Delta, R\right)$ некоторое почти $\Delta$-расслоение $\rho^{\prime \prime} \in[\rho]+\left[\rho^{\prime}\right]$ обладает набором функций перехода $\left\{\xi_{V U}^{\prime \prime}\right\}$, где $\xi_{V U}^{\prime \prime}=\xi_{V U}+\xi_{V U}^{\prime}$. При этом на пространстве расслоения $\rho^{\prime \prime}$ существует $G$-связность $H^{\prime \prime}$ с локальными формами связности $\left\{\omega_{U}^{\prime \prime} \equiv 0\right\}$. Но тогда для произвольной петли $x: I \rightarrow B$ и гомоморфизмов голономии $\tau_{H}, \tau_{H^{\prime}}$ и $\tau_{H^{\prime \prime}}$ имеют место формулы вида (4.4). Следовательно, $\tau_{H^{\prime \prime}}=\tau_{H}+\tau_{H^{\prime}}$ и $\eta_{0}\left(\left[\rho^{\prime \prime}\right]\right)=\eta_{0}([\rho])+\eta_{0}\left(\left[\rho^{\prime}\right]\right)$.

ПРЕДЛОЖЕНИЕ 4.5. Гомоморфизм $\eta_{0}$ сюрбективен. 
ДоказАтельство. Рассмотрим элемент $e=\tau+\operatorname{im} \operatorname{Exp}_{*}^{\Delta}$, где $\tau \in \operatorname{Hom}\left(H_{1}(B), T^{k}\right)$. Выберем точку $b \in B$ и покрытие $\mathscr{U}$ многообразия $B$ такое же, что и в предложении 3.1. Точно так, как в предложении 3.1 , для компонент связности $K(V U)$ пересечений $V \cap U$ множеств $U, V \in \mathscr{U}$ и точек $a \in K(V U)$ построим петли $x_{K(V U)}^{a}: I \rightarrow B$. Положим

$$
\xi_{V U}(a)=\tau\left(-\left[x_{K(V U)}^{a}\right]\right)
$$

По выбору покрытия $\mathscr{U}$ для любых $a, a^{\prime} \in K(V U)$ циклы $x_{K(V U)}^{a}$ и $x_{K(V U)}^{a^{\prime}}$ гомологичны. Следовательно, $\xi_{V U}: V \cap U \rightarrow T^{k}$-гладкое локально постоянное отображение. При этом для каждого $\delta \in \Delta$ разность $\xi_{V U} \circ R_{\delta}-\xi_{V U}$ также является локально постоянным отображением из $V \cap U$ в $T^{k}$.

Если $U, V, W \in \mathscr{U}$ и $a \in W \cap V \cap U$, то имеет место равенство (3.8). Поэтому существует почти $\Delta$-расслоение $\rho=(\xi, \mathscr{A})$ с атласом $\mathscr{A}(\mathscr{U}) \in \mathscr{A}$, имеющим систему функций перехода $\left\{\xi_{V U}\right\}$. Поскольку набор 1-форм $\left\{\omega_{U} \equiv 0\right\}$ удовлетворяет условию (3.5), на пространстве расслоения $\rho$ имеется $G$-связность $H$ с локальными формами связности $\left\{\omega_{U}\right\}$.

Рассмотрим петлю $x: I \rightarrow B$, разбиение $0=s_{0}<s_{1}<\cdots<s_{l}=1$ отрезка $I$ и компоненты связности $K U_{1}, \ldots, K U_{l}$ множеств из $\mathscr{U}$ такие, что $x\left(\left[s_{i-1}, s_{i}\right]\right) \subset K U_{i}$. Положим $a_{i}=x\left(s_{i}\right)$. Тогда по предложению 4.3 справедливо равенство (4.4). Отсюда в силу (4.7) следует, что

$$
\tau_{H}([x])=\tau\left(-\left[x_{U_{1} U_{l}}^{a_{l}}+x_{U_{l} U_{l-1}}^{a_{l-1}}+\cdots+x_{U_{2} U_{1}}^{a_{1}}\right]\right) .
$$

Поскольку петли $-\left(x_{U_{1} U_{l}}^{a_{l}}+x_{U_{l} U_{l-1}}^{a_{l-1}}+\cdots+x_{U_{2} U_{1}}^{a_{1}}\right)$ и $x$ гомологичны, (4.8) эквивалентно равенству $\tau_{H}([x])=\tau([x])$. Этим доказано, что $\tau_{H}=\tau$ и $\eta_{0}([\rho])=e$.

ПРЕДЛОЖЕНИЕ 4.6. Ядро ker $\eta_{0}$ состоит только из нейтрального әлемента əруппьь $\mathscr{B}\left(B, T^{k}, \Delta, R\right)$.

ДокАЗАТЕЛЬСтво. Пусть $\rho=(\xi, \mathscr{A})$ - почти $\Delta$-расслоение с проекцией $p: E \rightarrow B$, обладающее плоской $G$-связностью $H$ на его пространстве $E$, и $\eta_{0}([\rho])=0$. Тогда существует гомоморфизм $\bar{\tau} \in \operatorname{Hom}^{\Delta}\left(H_{1}(B), \mathbb{R}^{k}\right)$, удовлетворяющий равенству $\tau_{H}=$ $\operatorname{Exp~о\overline {\tau }.~В~силу~доказанной~вьше~леммы~ему~соответствует~такая~форма~} A \in \mathscr{Z}_{\Delta}^{1}\left(B, \mathbb{R}^{k}\right)$, что $I_{\Delta}\left([A]_{\Delta}\right)=\bar{\tau}$.

Рассмотрим форму $\omega$ связности $H$ и положим $\widehat{\omega}=\omega+p^{*} A$. Тогда по предложению 2.3 $\widehat{\omega}$ - форма некоторой $G$-связности $\widehat{H}$ на $E$. Согласно предложению 4.1 , построению формы $A$ и определению (4.2) $\tau_{\widehat{H}}=\tau_{H}-\operatorname{Exp} \circ I_{[A]}=\operatorname{Exp} \circ\left(\bar{\tau}-I_{[A]}\right)=0$. Таким образом, группа голономии $G$-связности $\widehat{H}$ тривиальна. Отсюда по предложению 2.4 вытекает, что $[\rho]=0$ в групе $\mathscr{B}\left(B, T^{k}, \Delta, R\right)$.

5. Основной результат. Примеры. Приложения. Пусть $\eta: \mathscr{B}\left(B, T^{k}, \Delta, R\right) \rightarrow$ $H_{\Delta}^{2}\left(B, \mathbb{R}^{k} \mid \mathbb{Z}^{k}\right)$ - гомоморфизм, построенный перед предложением 3.1 , im $\operatorname{Exp}_{*}^{\Delta}$ - образ естественного гомоморфизма $\operatorname{Exp}_{*}^{\Delta}: \operatorname{Hom}^{\Delta}\left(H_{1}(B), \mathbb{R}^{k}\right) \rightarrow \operatorname{Hom}\left(H_{1}(B), T^{k}\right)$, а $\eta_{0}$ : $\operatorname{ker} \eta \rightarrow \operatorname{Hom}\left(H_{1}(B), T^{k}\right) / \operatorname{im} \operatorname{Exp}_{*}^{\Delta}$ - гомоморфизм, определенный формулой (4.3). Согласно предложениям 4.4, 4.5 и $4.6 \eta_{0}$ - изоморфизм. Рассмотрим включение $\imath: \operatorname{ker} \eta \rightarrow$ $\mathscr{B}\left(B, T^{k}, \Delta, R\right)$ и положим $\zeta=\imath$ о $\eta_{0}^{-1}$. Тогда из предложения 3.1 следует, что справедлива 
ТЕОРема 5.1. Последовательность групп и гомоморфизмов

$$
0 \rightarrow \operatorname{Hom}\left(H_{1}(B), T^{k}\right) / \operatorname{im}_{\operatorname{Exp}_{*}^{\Delta}}^{\Delta} \stackrel{\zeta}{\rightarrow} \mathscr{B}\left(B, T^{k}, \Delta, R\right) \stackrel{\eta}{\rightarrow} H_{\Delta}^{2}\left(B, \mathbb{R}^{k} \mid \mathbb{Z}^{k}\right) \rightarrow 0
$$

точна и расщепляется. Поэтому

$$
\mathscr{B}\left(B, T^{k}, \Delta, R\right) \cong H_{\Delta}^{2}\left(B, \mathbb{R}^{k} \mid \mathbb{Z}^{k}\right) \oplus \operatorname{Hom}\left(H_{1}(B), T^{k}\right) / \operatorname{im}_{\operatorname{Exp}_{*}^{\Delta}}^{\Delta} .
$$

ПримеР 1. Рассмотрим сферу $B=S^{2}=\{r(u, v)=(\cos u \cos v, \cos u \sin v, \sin u) \mid$ $u \in[-\pi / 2, \pi / 2], v \in[0,2 \pi]\}$ и группы $T^{1}=\mathbb{R} / \mathbb{Z}$ и $\Delta=\mathbb{Z}_{n}$. Определим действие $R: B \times \Delta \rightarrow B$, полагая

$$
R_{[j]}(r(u, v))=r\left(u, v+\frac{2 \pi j}{n}\right)
$$

для $[j]=j+\mathbb{Z}, j=0,1, \ldots, n-1$. Геометрически $R_{[j]}$ есть поворот сферы на угол $2 \pi j / n$ вокруг прямой $(0,0) \times \mathbb{R}$. Пространство орбит $B / \Delta$ является орбиобразием с двумя коническими точками и гомеоморфно сфере $S^{2}$.

Так как преобразования $R_{[j]}$ сохраняют ориентацию, то они индуцируют тождественное преобразование группы $H_{2}(B)$. Поэтому $H_{\Delta}^{2}(B, \mathbb{R} \mid \mathbb{Z}) \cong H^{2}(B, \mathbb{Z}) \cong \mathbb{Z}$. В силу

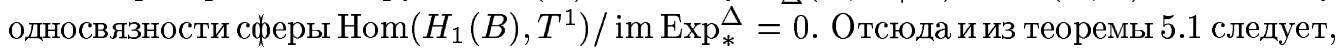
что $\mathscr{B}\left(B, T^{1}, \Delta, R\right) \cong \mathbb{Z} \cong \mathscr{B}\left(B, T^{1}\right)$, где $\mathscr{B}\left(B, T^{1}\right)$ - группа классов эквивалентности главных расслоений с базой $B$ и структурной групой $T^{1}$.

Как известно, произвольное такое расслоение имеет вид $\xi=m \chi$, где $m \in \mathbb{Z}$, а $\chi$ - pacслоение Хопфа. Простейший его атлас $\mathscr{A}(\mathscr{U})$ определяется покрытием $\mathscr{U}$, состоящим из множеств $U=\{r(u, v) \in B \mid u \in(-\pi / 4, \pi / 2]\}$ и $V=\{r(u, v) \in B \mid u \in[-\pi / 2, \pi / 4)\}$, а также функцией перехода $\xi_{V U}(r(u, v))=m v / 2 \pi+\mathbb{Z}$. Если точка $w=r(u, v)$ лежит в пересечении $E_{U} \cap E_{V}$, то согласно (5.1)

$$
\xi_{V U}\left(R_{[j]}(w)\right)=\frac{m}{2 \pi}\left(v+\frac{2 \pi j}{n}\right)+\mathbb{Z}=\frac{m v}{2 \pi}+\frac{m j}{n}+\mathbb{Z},
$$

при этом $\tau_{[j]}^{V U}(w)=\xi_{V U}\left(R_{[j]}(w)\right)-\xi_{V U}(w)=m j / n+\mathbb{Z}$.

Так как правая часть полученного равенства от точки $w$ не зависит, то $\mathscr{A}(\mathscr{U})$ - искомый почти $\Delta$-атлас. Согласно доказанному вьше любой другой почти $\Delta$-атлас расслоения $\xi$ эквивалентен $\mathscr{A}(\mathscr{U})$. С другой стороны, $\tau_{[j]}^{V U}=0$ в $T^{1}$ в том и только в том случае, если $m \equiv 0 \bmod n$.

Выводы. Для действия (5.1)

(1) главное расслоение $\xi=m \chi$ допускает (причем единственную) структуру почти $\Delta$-расслоения при любом $m \in \mathbb{Z}$;

(2) структурой, инвариантной относительно $\Delta$, оно обладает тогда и только тогда, когда характеристическое число т начело делится на порядок $n$ группь $\Delta$. 
ПримеР 2. Пусть теперь $B=T^{2}=(\mathbb{R} / \mathbb{Z})^{2}$ и $\Delta=\mathbb{Z}_{2}$. Действие $R: B \times \Delta \rightarrow B$ определим формулой

$$
R_{[j]}(x+\mathbb{Z}, y+\mathbb{Z})=\left((-1)^{j} x+\mathbb{Z},(-1)^{j} y+\mathbb{Z}\right) .
$$

При этом имеются четыре неподвижные точки $(s / 2+\mathbb{Z}, t / 2+\mathbb{Z}), s, t \in\{0,1\}$. Поэтому $B / \Delta$ - орбиобразие. Оно также гомеоморфно сфере $S^{2}$.

Так как $R_{[1]}$ сохраняет ориентацию тора, то $H_{\Delta}^{2}(B, \mathbb{R} \mid \mathbb{Z}) \cong \operatorname{Hom}^{\Delta}\left(H_{2}(B), \mathbb{Z}\right)=$ $\operatorname{Hom}\left(H_{2}(B), \mathbb{Z}\right) \cong \mathbb{Z}$. Вместе с тем $R_{[1]}$ меняет ориентации одномерных симплексов.

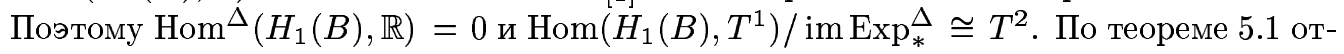
сюда следует, что $\mathscr{B}\left(B, T^{1}, \Delta, R\right) \cong \mathbb{Z} \oplus T^{2} \cong \mathscr{B}\left(B, T^{1}\right) \oplus T^{2}$. Таким образом, для действия (5.2) каждое главное расслоение с базой $B=T^{2}$ и структурной группой $T^{1}$ обладает бесконечным набором попарно не изоморфных почти $\Delta$-структур.

ПримеР 3. Рассмотрим, наконец, проективную плоскость $B=\mathbb{R} P^{2}$ и зададим действие той же группы $\Delta=\mathbb{Z}_{2}$ на $B$, полагая

$$
R_{[j]}(x: y: z)=\left((-1)^{j} x:(-1)^{j} y: z\right) .
$$

Неподвижными являются точки (0: $0: 1)$ и $(x: y: 0)$ при всех $x, y \in \mathbb{R}, B / \Delta \approx D^{2}$.

Очевидно, $H_{\Delta}^{2}(B, \mathbb{R} \mid \mathbb{Z})=0$. Поскольку $R_{[1]}$ переводит образующий элемент группы $H_{1}(B)$ в себя, то $\operatorname{Hom}^{\Delta}\left(H_{1}(B), \mathbb{R}\right)=\operatorname{Hom}\left(H_{1}(B), \mathbb{R}\right)$. Следовательно,

$$
\operatorname{Hom}\left(H_{1}(B), T^{1}\right) / \operatorname{im} \operatorname{Exp}_{*}^{\Delta}=\operatorname{Ext}\left(H_{1}(B), \mathbb{Z}\right) \cong \mathbb{Z}_{2} .
$$

В результате $\mathscr{B}\left(B, T^{1}, \Delta, R\right) \cong \mathbb{Z}_{2} \cong \mathscr{B}\left(B, T^{1}\right)$. Это значит, что каждое из двух (не изоморфных) главных расслоений с базой $B=\mathbb{R} P^{2}$ и структурной группой $T^{1}$ допускает (при действии (5.3)) почти $\Delta$-структуру, но только одну.

Пусть далее $g$ - риманова (лоренцева) метрика, $f$-замкнутая 2-форма и $u$-гладкая функция на многообразии $B$. Четверку $\Gamma=(B, g, f, u)$ принято называть гироскопической системой. Предположим, что выполнены условия

$$
f=\theta \circ F, \quad \text { где } \quad \theta \in \operatorname{Hom}\left(\mathbb{R}^{k}, \mathbb{R}\right) \quad \text { и } \quad[F] \in H^{2}\left(B, \mathbb{R}^{k} \mid \mathbb{Z}^{k}\right), \quad u>0 .
$$

Тогда сушествует главное расслоение $\xi$ с проекцией $p: E \rightarrow B$, структурной группой $T^{k}$ и характеристическим классом $[F]$. На его пространстве $E$ найдется $T^{k}$-связность $H$ с формой связности $\omega$ и формой кривизны $d \omega=p^{*} F$. Полагая

$$
\bar{g}=\frac{|\theta|^{2}}{2 u \circ p}\langle\omega, \omega\rangle+p^{*} g,
$$

где $\langle\cdot, \cdot\rangle$ - стандартная евклидова метрика на $\mathbb{R}^{k}$, можно построить риманову (лоренцеву) метрику $\bar{g}$ на $E$. Любое движение системы $\Gamma$ представляет собой проекцию подходящим образом выбранной геодезической многообразия $(E, \bar{g})[2],[3]$.

Допустим, что система $Г$ инвариантна относительно действия $R: B \times \Delta \rightarrow B$ конечной группы $\Delta$. Легко видеть, что при этом форму $F$ также можно выбрать инвариантной. 
Действительно, если $f=\hat{\theta} \circ \widehat{F}$, где форма $\widehat{F}$ удовлетворяет условию $\operatorname{im} I_{[\widehat{F}]} \subset \mathbb{Z}^{k}$, но не инвариантна относительно $R$, то положим $\theta=(1 /|\Delta|) \hat{\theta}$ и $F=\sum_{\delta \in \Delta} R_{\delta}^{*} \widehat{F}$. Тогда $\theta \circ F=(1 /|\Delta|) \sum_{\delta \in \Delta} R_{\delta}^{*} f=f$ и $I_{[F]}=\sum_{\delta \in \Delta} I_{R_{\delta}^{*} \widehat{F}}=\sum_{\delta \in \Delta} I_{\widehat{F}} \circ R_{\delta}$. Следовательно, $\operatorname{im} I_{[F]} \subset \mathbb{Z}^{k}$. По построению $R_{\delta}^{*} F=F$ для всех $\delta \in \Delta$.

Согласно предложению 2.2 при инвариантности формы $F$ расслоение $\xi$ обладает почти $\Delta$-структурой $\mathscr{A}$, а $H$ является $G$-связностью, $G=\Delta \times T^{k}$. В этой ситуации метрика $\bar{g}$ инвариантна как относительно действия $S: E \times T^{k} \rightarrow E$ структурной группы $T^{k}$, так и относительно соответствующего $\mathscr{A}$ многозначного действия $\mathscr{R}$ группы $\Delta$.

Пусть теперь на пространстве $E$ почти $\Delta$-расслоения $\rho=(\xi, \mathscr{A})$ задана инвариантная относительно $S$ и $\mathscr{R}$ риманова (лоренцева) метрика $\bar{g}$. Тогда ортогональные слоям касательные векторы к $E$ образуют $G$-связность $H$. С ее помошью метрика $\bar{g}$ легко приводится к виду (5.5). Это значит, в частности, что она индуцирует гомоморфизм $\theta: \mathbb{R}^{k} \rightarrow \mathbb{R}$, а также метрику $g$, форму $F$ и функцию $u$ на базе $B$. Полагая $f=\theta \circ F$, получим гироскопическую систему $\Gamma=(B, g, f, u)$, удовлетворяющую условиям (5.4) и инвариантную относительно $R$.

Выводы.

(1) Каждая гироскопическая система Г на многообразии В, обладающая свойствами (5.4) и инвариантная относительно действия $R: B \times \Delta \rightarrow B$, порождает почти $\Delta$-расслоение $\rho$ и инвариантную относительно действия $S: E \times T^{k} \rightarrow E$ структурной группь $T^{k}$ и многозначного действия $\mathscr{R}$ группы $\Delta$ риманову (лоренцеву) метрику $\bar{g}$ на его пространстве Е. При этом динамика системы Г моделируется геодезическими многообразия $(E, \bar{g})$.

(2) Верно и обратное. Любое почти $\Delta$-расслоение $\rho$ и инвариантная относительно $S$ и $\mathscr{R}$ риманова (лоренчева) метрика $\bar{g}$ на его пространстве могут быть получены таким образом с помощью некоторой инвариантной гироскопической системы на базе $B$.

\section{СПИСОК ЦИТИРОВАННОЙ ЛИТЕРАТУРЫ}

[1] Новиков С. П. Гамильтонов формализм и многозначный аналог теории Морса // УМН. 1982. Т. 37. № 5. C. 3-49.

[2] Яковлев Е.И. Геодезическое моделирование и условия разрешимости двухконцевой задачи для многозначных функционалов // Функцион. анализ и его прилож. 1996. Т. 30. № 1. С. 89-92.

[3] Яковлев Е.И. О существовании решений двухточечных краевых задач для гироскопических систем релятивистского типа // Алгебра и анализ. 1997. Т. 9. № 2. С. 256-271.

[4] Яковлев Е. И. Почти главные расслоения // Матем. сб. 1999. Т. 190. №9. С. 151-176.

[5] Бредон Г. Введение в теорию компактных групп преобразований. М.: Наука, 1980.

[6] Хьюзмоллер Д. Расслоенные пространства. М.: Мир, 1970.

[7] Kobayashi S. Principal fibre bundles with the 1-dimensional toroidal group // Tôhoku Math. J. 1956. V. 8. P. 29-45.

[8] Чжэнь Шэн-Шэнь. Комплексные многообразия. М.: ИЛ, 1961. 